\title{
RAMIFIED COVERINGS AND GAUSSIAN MAPS OF SMOOTH ALGEBRAIC CURVES
}

\author{
E. BALlico* AND C. KeEM ${ }^{\dagger}$
}

\begin{abstract}
We show the existence of special curves which are ramified coverings of irrational curves with surjective Wahl maps. We also show the failure of a key property of Gaussian maps in positive characteristic, i.e. the Gherardelli's lower bound for the rank of a Gaussian map.
\end{abstract}

\section{Introduction}

In the last couple of decades, many works have been done to clarify the structure (and in particular the rank) of certain linear maps, called Gaussian maps. These maps capture many geometric informations on the geometry of projective varieties, on their differential geometric properties and on their deformation theoretic properties. We do not recall here the definitions and the main properties of them (for instance, the reader can see both in [7] or [11]). For very good reasons, one particular Gaussian map is called Wahl map (see [10]).

We fix notations. We always work over an algebraically closed base field $\mathbf{K}$ with char $\mathbf{K} \neq 2$. Let $X$ be a projective variety, $L$ and $M$ line bundles on $X, V$ a subspace of $H^{0}(X, L), W$ a subspace of $H^{0}(X, M)$. The Gaussian map of the pair $(V, W)$ is a linear map

$$
\Psi_{V, W}: V \otimes W \rightarrow H^{0}\left(X, \Omega_{X}^{1} \otimes L \otimes M\right) .
$$

If $V=W$ we will write $\Psi_{V}$ instead of $\Psi_{V, V}$. The map $\Psi_{V}$ is uniquely determined by its restriction (denoted in the same way) to $\Lambda^{2}(V)$. If $V=H^{0}(X, L)$ and $W=H^{0}(X, M)$ we will use the notations $\Psi_{L, M}$ and $\Psi_{L}$ instead of $\Psi_{V, W}$ and $\Psi_{V}$. If $K:=K_{X}$ (the canonical sheaf) is a line bundle, the Gaussian map $\Psi_{K}$ is called the Wahl map of $X$. Note that if $X$ is a smooth curve the Wahl map $\Psi_{K}$ of $X$ is a linear map from $\Lambda^{2}\left(X, K_{X}\right)$ to $H^{0}\left(X, K_{X}^{\otimes 3}\right)$.

In the first part of this paper we will prove the following result.

2000 Mathematics Subject Classifications: 14H10, 14H45, 14J25, 14 J26.

Key words: algebraic curves, Gaussian maps, ramified covering, ruled surfaces.

* Partially supported by MIUR and GNSAGA of INdAM (Italy).

† Supported by Korea Research Foundation \#2004-015-C00015.

Received March 16, 2004; revised October 12, 2004. 
THEOREm 0.1. Assume $\operatorname{char}(\mathbf{K})=0$ or $\operatorname{char}(\mathbf{K})=p>2$. Then there exists an integer $g_{0}$ such that the following statement holds. For every even (resp. odd) integer $q \geq 2$, for every even (resp. odd) integer $g \geq g_{0}$ and for every smooth curve $C$ of genus $q$ there is a smooth curve $X$ of genus $g$ which is a 5:1 covering of $C$ whose Wahl map $\Psi_{K}$ is surjective.

Taking a hyperelliptic curve as the curve $C$ in the statement of Theorem 0.1, we obtain the following corollary related to [11], $\$ 4$ and [4], Problem 8.5.

Corollary 0.2. Assume $\operatorname{char}(\mathbf{K})=0$ or $\operatorname{char}(\mathbf{K})=p>2$. There exists an integer $g_{0}$ such that for every $g \geq g_{0}$ a general 10-gonal curve of genus $g$ has a surjective Wahl map.

We remark that Corollary 0.2 is a very special case of [6], Theorem 1.2 (at least in characteristic zero). We further remark that Proposition 4.1 of [6] shows that the Wahl map of a double or triple covering is not surjective.

Theorem 0.1 will be proved in section 1 as a corollary to the Theorem 1.1 and Theorem 1.2. These theorems and their proofs are related to [12]; Theorem 4.11, Lemma 4.12 and Corollary 4.13, [4]; Ch. 3 and problem 8.5, and [5]. Indeed, in characteristic 0 case it would be possible to prove Corollary 0.2 and a slightly weaker form of Theorem 0.1 just using [12]; Theorem 4.11, Lemma 4.12 and proof of Corollary 4.13. The main point is not Corollary 0.2 but rather that the surjectivity of the Wahl map also holds true for some very special (e.g. 10gonal) curves of sufficiently high genus.

In the second (and last) section of this paper we show the failure of a key property of Gaussian maps in positive characteristic: Gherardelli's lower bound for the rank of a Gaussian map (see Corollary 2.2 and Example 2.4).

\section{Proofs of 0.1 and 0.2}

In this section we prove Theorem 0.1 and Corollary 0.2 as a consequence of Theorem 1.1 and Theorem 1.2. Here are the notations we will use in this section.

We fix a smooth curve $C$ of genus $q \geq 2$ and a rank 2 normalized vector bundle $E$ on $C$. Set $S:=\mathbf{P}(E)$ and let $\pi: S \rightarrow C$ be the associated projection. Let $-e$ be the minimal degree of the self-intersection of a section of $\pi$; by a theorem of Nagata we have $e \geq-q ; E$ is stable if and only if $e<0$. We work on $\operatorname{Pic}(S)$ modulo numerical equivalence (which will be denoted by $\approx$ ). We take as basis of $\operatorname{Pic}(S)$ modulo numerical equivalence the class $f$ of a fiber of $\pi$ and the class $h$ of a minimal degree section of $\pi$. Hence $h^{2}=-e, h . f=1$ and $f^{2}=0$; we will use the additive notations for $\operatorname{Pic}(S) / \approx$. We fix integers $a, b$ with $a>0, b>e a$ if $e \geq 0, b>0$ if $e<0$ and a smooth curve $X$ of type $a h+b f$. We have

$$
K_{S} \approx-2 h+(2 q-2-e) f, \quad K_{X}=\left(K_{S}+X\right)_{\mid X}
$$

and 


$$
p_{a}(X)=1+a b-\left(e a^{2} / 2\right)+(e a / 2)+q a-a-b
$$

by the adjunction formula. Set $K:=K_{S}$. Note that for every line bundle $L$ on $S$ (and there is a $q$-dimensional family of them) whose numerical class is $a h+b f$, we have

$$
\begin{aligned}
h^{0}(S, L) & =1-q+\left(L \cdot\left(L-K_{S}\right) / 2\right) \\
& =1-q+((a h+b f)((a+2) h+(b+e+2-2 q) f) / 2) \\
& =1-q-a q+a b-(e a / 2)-\left(e a^{2} / 2\right)+a+b
\end{aligned}
$$

by Riemann-Roch formula and vanishing theorems. Note that every curve $X$ we will use will appear only once and for a unique such line bundle just because for genus reasons any such $X$ can be a $5: 1$ covering of another curve in at most one way. Hence indeed we will find a family of large dimension (diverging with $g$ ) satisfying the thesis of Theorem 0.1 (for $a=5$ and taking for instance $e=0$ or $e=1$ ). We use the following commutative diagram considered in many of the quoted references.

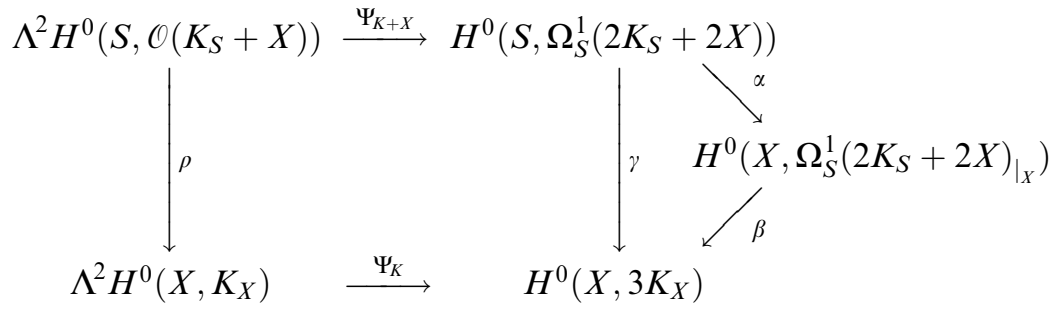

Here the restriction map $\rho$ is not surjective because $q>0$; we will call $\Psi_{K} \circ \rho$ the relative Wahl map of $X$ (or of the pair $(X, S)$ ). To prove the surjectivity of $\Psi_{K}$, it is sufficient to prove the surjectivity of the relative Wahl map which follows if we have the surjectivity of $\Psi_{K+X}$ and $\gamma$. Even just the surjectivity of $\gamma$ (e.g. the surjectivity of $\alpha$ and $\beta)$ is interesting since it shows that $\operatorname{dim}\left(\operatorname{coker}\left(\Psi_{K} \circ \rho\right)\right) \leq$ $\operatorname{dim}\left(\operatorname{coker}\left(\Psi_{K+X}\right)\right)$. It is the part which fails if $q=0$.

THEOREM 1.1. With the notations just introduced we have:

(i) $\beta$ is surjective.

(ii) There is a function $B(q, e, a)$ (depending only on the integers $q, e, a)$ such that if $b \geq B(q, e, a)$ the map $\alpha$ is surjective.

Proof. (i): By the conormal exact sequence of $X$

$$
0 \rightarrow \mathcal{O}_{X}\left(2 K_{S}+X\right) \rightarrow \mathcal{O}_{S} \otimes \Omega_{S}^{1}\left(2 K_{S}+2 X\right) \rightarrow K_{X}^{\otimes 3} \rightarrow 0,
$$

$\operatorname{coker}(\beta)$ is contained in $H^{1}\left(X, \mathcal{O}_{X}\left(2 K_{S}+X\right)\right)$ which is dual to $H^{0}\left(X,\left(-K_{S}\right)_{\mid X}\right)$. From the exact sequence on $S$ :

$$
0 \rightarrow \mathcal{O}_{S}\left(-K_{S}-X\right) \rightarrow \mathcal{O}_{S}\left(-K_{S}\right) \rightarrow \mathcal{O}_{S}\left(-K_{S}\right)_{\mid X} \rightarrow 0
$$

and the fact that for $q \geq 2$ we have $h^{0}\left(S,-K_{S}\right)=0$, we see that it is sufficient to prove that $H^{1}\left(S,-K_{S}-X\right)=0$. By a vanishing theorem of Kodaira (in 
positive characteristic we have to use that Kodaira vanishing holds for surfaces of Kodaira dimension $<0$ in [8]; Ch. II, Theorem 1.6 at p. 125); in positive characteristic to have the ampleness of $K_{S}+X$ use [9], Ch. V, Ex. 2.14 and note that $\left(K_{S}+X\right)^{2}>0$ and $\left(K_{S}+X\right) \cdot h>0$ for $e>0$.

(ii): By the following exact sequence on $S$,

$$
0 \rightarrow \Omega_{S}^{1}\left(2 K_{S}+X\right) \rightarrow \Omega_{S}^{1}\left(2 K_{S}+2 X\right) \rightarrow \Omega_{S}^{1}\left(2 K_{S}+2 X\right)_{\mid X} \rightarrow 0
$$

to prove the surjectivity of $\alpha$ it is enough to show the vanishing of the space $H^{1}\left(S, \Omega_{S}^{1}\left(2 K_{S}+X\right)\right)$. This vanishing depends only on the line bundle $\mathcal{O}_{S}(X)$, not on the choice of the curve $X$ in the linear system $|X|$ on $S$. Let $F$ be any fiber of $\pi$, say $F=\pi^{-1}(P)$. Hence $F \cong \mathbf{P}^{1}$ and $\mathcal{O}_{S}(-F)_{\mid F} \cong \mathcal{O}_{F}$. From the conormal bundle exact sequence of the inclusion of $F$ in $S$

$$
0 \rightarrow \mathcal{O}_{S}(-F)_{\mid F} \rightarrow \Omega_{S \mid F}^{1} \rightarrow \Omega_{F}^{1} \rightarrow 0,
$$

we obtain that $\Omega_{S \mid F}^{1}$ is a direct sum of a line bundle of degree 0 and a line bundle of degree -2 . Hence $\Omega_{S}^{1}\left(2 K_{S}+X\right)_{\mid F}$ is a direct sum of a line bundle of degree $a-4$ and a line bundle of degree $a-6$. Hence if $a \geq 5$ we have $h^{1}\left(F, \Omega_{S}^{1}\left(2 K_{S}+X\right)_{\mid F}\right)=0, h^{0}\left(F, \Omega_{S}^{1}\left(2 K_{S}+X\right)_{\mid F}\right)=2 a-8$ and hence $R^{1} \pi_{*}\left(\Omega_{S}^{1}\left(2 K_{S}+X\right)\right)=0$, while $R^{0} \pi_{*}\left(\Omega_{S}^{1}\left(2 K_{S}+X\right)\right)$ is a rank $2 a-8$ vector bundle on $C$. By the Leray spectral sequence of $\pi$ it is sufficient to show that $H^{1}\left(C, R^{0} \pi_{*}\left(\Omega_{S}^{1}\left(2 K_{S}+X\right)\right)\right)=0$. Take any integer $c \geq 0$ such that $|X-c F| \neq \emptyset$ and fix $Y \in|X-c F|$. We have

$$
\Omega_{S}^{1}\left(2 K_{S}+X\right) \cong \Omega_{S}^{1}\left(2 K_{S}+Y\right) \otimes \mathcal{O}_{S}(c F),
$$

and hence

$$
R^{0} \pi_{*}\left(\Omega_{S}^{1}\left(2 K_{S}+X\right)\right) \cong R^{0} \pi_{*}\left(\Omega_{S}^{1}\left(2 K_{S}+Y\right)\right) \otimes \mathcal{O}_{C}(c P)
$$

by the projection formula. For any fixed $Y$ the vector bundle $R^{0} \pi_{*}\left(\Omega_{S}^{1}\left(2 K_{S}+Y\right)\right)$ is a fixed vector bundle on $C$ and hence $H^{1}(C$, $\left.R^{0} \pi_{*}\left(\Omega_{S}^{1}\left(2 K_{S}+Y\right)\right) \otimes \mathcal{O}_{C}(c P)\right)=0$ for $c \gg 0$, say for all $c \geq c_{0}$. The minimal integer $c_{0}$ depends only on the integer $q$ and the numerical invariants of the Harder-Narashiman filtration of the vector bundle $R^{0} \pi_{*}\left(\Omega_{S}^{1}\left(2 K_{S}+Y\right)\right.$ ) (ranks and degrees of its subquotients). These numerical invariants depend only on the numerical invariants of $S$ (i.e. from $q$ and $e$ ) and the numerical invariants of $Y$, say $a$ and $b^{\prime}$. Furthermore, there is one $b^{\prime}$ (at least) for any fixed $q, e, a$. Hence $H^{1}\left(C, R^{0} \pi_{*}\left(\Omega_{S}^{1}\left(2 K_{S}+X\right)\right)\right)=0$ for fixed $q, e, a$ if $b=b^{\prime}+c$ is large, say for all $b \geq B(q, e, a)$.

We were unable to give an explicit form for the function $B(q, e, a)$ in part (ii) of Theorem 1.1.

THEOREM 1.2. There is a computable function $D(q, e, a)$ depending only on $q$, $e$, $a$, such that if $b \geq D(q, e, a)$, then $\Psi_{K+X}$ is surjective.

Proof. Several proofs (each with a different estimate for the function $D(q, e, a))$ seems to be available; some of these proofs use different interpretations 
of the Gaussian maps. For the case $e=0$, see Remark 1.3; for the general case one can use also either the method of [3] as in [2] or a Leray spectral sequence and the interpretation in terms of the diagonal of $S \times S$ given in [5]. Here we use the interpretation of the Gaussian map given in [11], Lemma 1.7. Set $L(b):=K_{S}+X$ (as a function of $b$ for fixed $q, e, a$ ). First consider (restricting to a fiber $F$ of $\pi$ ) what happens if instead of the curve $X$ we take a curve $X^{\prime}$ numerically equivalent to $a h+(b+1) f$ in the linear system $\mathcal{O}_{S}(X) \otimes \mathcal{O}_{S}(F)$. Consider the restriction of the data to $F$. Note that $L(b)_{\mid F}$ is a line bundle of degree $a-2>0$ on $\mathbf{P}^{1}$. Take another integer $b^{\prime}$ and consider $\Psi_{L(b), L\left(b^{\prime}\right)}$. First note that with the notations of [11], Lemma 1.7, we need to prove $h^{1}\left(S, M_{L(b)} \otimes\right.$ $L(b))=0$. First, restricting to $F$, we obtain that for large $b$ (say $b \geq b_{0}(q, e, a)$ ) the bundle $M_{L(b)} \otimes L(b)$ is spanned. Then we see that $h^{1}\left(S, M_{L(b)} \otimes L(b)\right)$ for large $b$ is a non increasing function of $b$ (up to now this would follow also from a Leray spectral sequence and the interpretation in terms of the diagonal of $S \times S$ ). Then we show that if $b^{\prime}>b$ we have

$$
h^{1}\left(S, M_{L(b)} \otimes L(b)\right) \leq h^{1}\left(S, M_{L\left(b^{\prime}\right)} \otimes L\left(b^{\prime}\right)\right)
$$

and that if $b^{\prime \prime}>b^{\prime}>b \geq b_{0}(q, e, a)$, then

$$
h^{1}\left(S, M_{L(b)} \otimes L\left(b^{\prime \prime}\right)\right) \leq \max \left(0, h^{1}\left(S, M^{L(b)} \otimes L\left(b^{\prime}\right)\right)-1\right)
$$

(restricting to the fiber $F$, using the Leray spectral sequence of $\pi$, the projection formula and using the fact that for every sheaf $U$ on $C$ and every $P \in C$, we have $h^{1}\left(C, U \otimes \mathcal{O}_{C}(P)\right)<h^{1}(C, U)$ if $\left.h^{1}(C, U)>0\right)$.

Proof of Theorem 0.1. Set

$$
G(q, e, a, b):=p_{a}(X)=1+a b-\left(e a^{2} / 2\right)+(e a / 2)+q a-a-b .
$$

Note that $G(q, e, a, b+1)=G(q, e, a, b)+a-1$. Hence fixing $q$, $e$ and taking $a=5$, if for one large $b$ we cover a genus $g^{\prime}$, then we cover all the genera $g^{\prime}+4 t$ with $t$ positive integer. Then note that $G(q, e+1, a, b)=G(q, e, a, b)-a^{2} / 2+$ $a / 2$. Hence, since $\operatorname{gcd}(4,10)=2$ we may use the same trick taking two consecutive integers instead of the integer $e$ and conclude the proof of 0.1 .

Remark 1.3. To use only the case $S=\mathbf{P}^{1} \times C$ as in [12], Theorem 4.11, Lemma 4.12 and Corollary 4.13 (which would give explicit and very good bounds), just take instead of $C, 4$ curves $C_{i}, 1 \leq i \leq 4$, with $p_{a}\left(C_{i}\right)=q+i-1$ and note that $G(q+1, e, a, b)=G(q, e, a, b)+a$ and that 5 and 4 are coprime. In this way, we also get Corollary 0.2 with a more explicit bound on $g_{0}$.

\section{The failure of Gherardelli's bound in characteristic $p$}

In this section we will show the failure in positive characteristic of a key property of the Gaussian map for non complete linear series, i.e. Gherardelli's lower bound of the rank of every Gaussian map (Theorem 2.1 and Corollary 2.2). Nevertheless, the more interesting applications of Gherardell's bound hold in positive characteristic (see Proposition 2.3). The key point of this section is Example 2.4. 
Theorem 2.1. Fix integers $n$ and $p$ with $n \geq 2$, $p$ prime and $p \neq 2$. Set $x:=$ $[(n-1) / p] . \quad$ Assume $\operatorname{char}(\mathbf{K})=p$. Let $C$ be a rational normal curve in $\mathbf{P}^{n}$. Then there exists a linear subspace $V$ of $\mathbf{P}^{n}$ with $\operatorname{dim}(V)=x$ and such that every tangent line to $C$ intersects $V$. Furthermore, no integer smaller than $x$ has this property.

Proof. Recall that a curve $Z$ in a projective space $M$ is said to be strange if there is a point $P \in M$ (called the strange point of $Z$ ) such that every tangent line to a smooth point of $Z$ contains $P$. Let $y$ be the minimal integer such that there a rational (but singular) strange curve $D$ in $\mathbf{P}^{y}$ (hence $D$ is the image of a projection $p_{W}$ of $C$ from a linear space $W$ of dimension $n-y-1$ with $W \cap C=$ $\emptyset$. Let $V$ be the $(n-y)$-dimensional linear subspace of $\mathbf{P}^{n}$ containing $W$ and mapped to $v$ by the projection $p_{W}$. Since every tangent line to $D_{r e d}$ contains $v$, every tangent line to $C$ intersects $V$. Since the projection $p_{v}$ of $D$ from $v$ into $\mathbf{P}^{y-1}$ is not separable and its image is a non degenerate curve, we have $n=$ $\operatorname{deg}(D) \geq p\left(\operatorname{deg}\left(D^{\prime}\right)\right) \geq p(y-1)$. Hence $y \leq x+1$. To prove the theorem it is sufficient to use that in [1] it is proved the existence of a rational strange curve of degree $p x$ in $\mathbf{P}^{x+1}, D$ lying on a cone $T$ with vertex $v$ and with as base a rational normal curve $D^{\prime}$ of $\mathbf{P}^{y-1}, D$ with $v$ as strange point.

Taking duals, the following statement is equivalent to Theorem 2.1 and shows in what sense Gherardelli's lower bound fails in positive characteristic (compare with [10], (1.4)).

Corollary 2.2. Assume $\operatorname{char}(\mathbf{K})=p>2$. Let $L$ be a line bundle of degree $n$ on $\mathbf{P}^{1}$ and $\Psi_{L}$ the associated Gaussian map. Then the set of decomposable vectors of $\Lambda^{2} H^{0}\left(\mathbf{P}^{1}, L\right)$ intersects $\operatorname{Ker}\left(\Psi_{L}\right)$ in a set of dimension $x-1$ with $x:=\left[\frac{n-1}{p}\right]$.

However, we still have the following result as in characteristic 0 .

Proposition 2.3. Assume char $(\mathbf{K}) \neq 2$. The Gaussian map $\Psi_{L}$ of the degree $n$ line bundle $L$ on $\mathbf{P}^{1}$ has rank $2 n-1$. Hence the Wahl map of the canonical bundle of a hyperelliptic curve of genus $g$ has rank $2 g-3$.

Proof. Note (as in [6], first sentence of section 1, or [11], Theorem 4.4) that for the base $\left\{x^{i}\right\}_{0 \leq i \leq n}$ of $H^{0}\left(\mathbf{P}^{1}, L\right)$ we have

$$
\Psi_{L}\left(x^{i} \wedge x^{j}\right)=(j-i) x^{i+j-1} d x, \quad 0 \leq i<j \leq n .
$$

It is easy to check that for every integer $u$ with $1 \leq u \leq 2 n-1$ we may find two integers $i$ and $j, i \neq j$, with $j-i$ not a multiple of $p:=\operatorname{char}(\mathbf{K})>2$ and $i+j=u$.

The main point of this section is that the failure of the classical lower bound in Gherardelli's theorem is reflected in the failure of a Gaussian map for certain non complete linear systems, as shown by the following example. 
Example 2.4. Assume char $(\mathbf{K})=p>2$. For every integer $n$, a with $2 \leq$ $a \leq n-p$ there is a base point free linear subspace $V$ of $H^{0}\left(\mathbf{P}^{1}, \mathcal{O}(n)\right)$ with $\operatorname{dim}(V)=a+1$ and such that $\operatorname{rank}\left(\Psi_{V}\right)<2 a-1$.

Proof. Take a base $\left\{x^{i}\right\}_{0 \leq i \leq n}$ of $H^{0}\left(\mathbf{P}^{1}, \mathcal{O}(n)\right)$ with $d x^{i}=i x^{i-1} d x$. Take a subspace $V$ of dimension $a+1$ spanned by elements $\left\{x^{i}\right\}_{i \in J}$ of this base, $J=\left\{j_{1}, \ldots j_{a+1}\right\}, \quad j_{1}<\cdots<j_{a+1}$ with, say, $p+j_{a}=j_{a+1}$. Then we have $\Psi_{V}\left(x^{j_{a}} \wedge x^{j_{a+1}}\right)=0$.

\section{REFERENCES}

[1] E. Ballico, On strange projective curves, Rev. Roumaine Math. Pures Appl. 37 (1992), $741-745$.

[2] E. Ballico and C. Ciliberto, On Gaussian maps for projective varieties, Geometry of complex projective varieties (Cetraro, 1990), Mediterranean Press (Rende) Sem. Conf. 9 (1993), 9-33.

[ 3 ] A. Beauville and J.-Y. Merindol, Sections hyperplanes des surfaces K-3, Duke Math. J. 55 (1987), 873-878.

[4] J. Brawner, The Gaussian map $\Psi_{K}$ for curves with special linear series, Ph.D. Thesis, Univ. North Carolina (1992).

[ 5 ] C. Ciliberto, J. Harris and R. Miranda, On the surjectivity of the Wahl map, Duke Math. J. 57 (1988), 829-858.

[6] C. Ciliberto And A. F. Lopez, On the number of moduli of extendable canonical curves, Nagoya Math. J. 167 (2002), 101-115.

[7] C. Ciliberto and R. Miranda, Gaussian maps for certain families of canonical curves, Complex projective geometry, London Math. Soc. Lecture Notes Series (Cambridge University Press) 179 (1992), 106-127.

[8] T. Ekedhal, Canonical models of surfaces of general type in positive characteristic, Publ. I.E.H.S. 67 (1988), 97-144.

[9] R. Hartshorne, Algebraic Geometry, Springer-Verlag, New York, 1977.

[10] J. WAHL, The Jacobian algebra of a graded Gorenstein singularity, Duke Math. J. 55 (1986), 843-871.

[11] J. WAHL, Introduction to Gaussian maps on an algebraic curve, Complex projective geometry, London Math. Soc. Lecture Notes Series (Cambridge University Press) 179 (1992), 304-323.

[12] J. WAHL, Gaussian maps on algebraic curves, J. Diff. Geom. 32 (1990), 77-98.

Department of Mathematics

UNIVERSITÀ DI TRENTO

38050 Povo(TN), Italy

fax: Italy +0461881624

e-mail: ballico@science.unitn.it

Department of Mathematics

College of Natural Sciences

SeOUl NaTional UNiversity

Seoul 151-742, South Korea

fax: Korea+28806549

e-mail: ckeem@math.snu.ac.kr 